\title{
Fenómeno de no reflujo en la angioplastia coronaria: incidencia, características clínico-angiográficas y eficacia del tratamiento
}

\author{
Dres. Rodolfo Gutiérrez, Gustavo Vignolo, Ignacio Batista, Rodrigo Abreu, \\ Santiago Alonso, Pablo Vázquez, Mariana Dieste, Jorge Mayol, Tomás Dieste
}

\begin{abstract}
Resumen gráficas, terapéuticas y pronósticas. $1,1 \%, \mathrm{p}=0,017)$ y nueva ATC ( $19 \%$ vs $1,1 \%, \mathrm{p}=0,009$ ). mejorar significativamente el flujo coronario.

Palabras clave: $\quad$ FENÓMENO DE NO REFLUJO

ANGIOPLASTIA CORONARIA

ADENOSINA

TIMI FRAME COUNT
\end{abstract}

Introducción: el fenómeno de no reflujo (NR) coronario se define como la persistencia de un flujo inadecuado menor a TIMI 3 durante la angioplastia coronaria (ATC), en ausencia de obstáculo macroscópico en las arterias coronarias epicárdicas. Objetivo principal: determinar la incidencia de NR en pacientes tratados con ATC por cualquier indicación. Objetivos secundarios: describir las características clínicas y angiográficas de la población con NR, características del procedimiento de angioplastia, así como el tratamiento y el valor pronóstico del NR.

Métodos: estudio observacional, prospectivo, unicéntrico, sobre el total de ATC realizadas en un centro de cardiología intervencionista entre octubre de 2016 y enero de 2017, excluyendo la reestenosis intrastent y la enfermedad de injertos venosos. Se definieron dos grupos: control (flujo normal) y con fenómeno de NR. Se analizaron variables clínicas, angio-

Resultados: se incluyó un total de 322 pacientes, 291 control y 31 NR. La incidencia de NR fue de 9,6\%, presentándose en 20,7\% de los síndromes coronarios agudos con elevación de ST (SCAcST), en 6,3\% de los síndromes coronarios agudos sin elevación de ST (SCAsST) y en 3\% de las anginas estables. La adenosina a dosis altas fue el tratamiento más utilizado. Se comprobó una mejoría significativa del flujo coronario evaluado por TIMI Frame Count asociado al tratamiento instituido ( $39,4 \pm 1,6$ pretratamiento vs $21,9 \pm 1,2$ postratamiento, $\mathrm{p}<0,001)$. A tres meses, en los pacientes con NR vesus control se observó una mayor incidencia de ángor $(6,6 \%$ vs $1,1 \%$ respectivamente, $\mathrm{p}=0,001)$, nueva coronariografía ( $10 \%$ vs

Conclusiones: la incidencia de NR en pacientes sometidos a ATC fue de 9,6\%, presentándose más frecuentemente en el SCAcST. La adenosina intracoronaria a dosis altas fue el tratamiento más frecuentemente instituido y fue eficaz para

Centro Cardiológico Americano, Sanatorio Americano, FEPREMI (Federación de Prestadores Médicos del Interior).

Correspondencia: Dr. Rodolfo Gutiérrez Bartlett. Diagonal 6 17-87 Condominio Broken Sound, Zona 10 Guatemala, Guatemala. Correo electrónico: gutierrezrodolfo83@gmail.com

Los autores declaran no tener conflictos de intereses.

Recibido: Mar 23, 2018; aceptado: Jun 14, 2018 


\section{No-reflow phenomenon in coronary angioplasty: incidence, clinical-angiographic characteristics and efficacy of treatment}

\section{Summary}

Introduction: no-reflow phenomenon is defined as persistence of inadequate flow less than TIMI 3 during coronary angioplasty in the absence of an obstacle in epicardial coronary arteries. Primary endpoint: to determine the incidence of no-reflow in patients treated with coronary angioplasty performed for any indication. Secondary endpoints: to describe the clinical and angiographic characteristics, procedural characteristics, treatment and prognostic value of no-reflow phenomenon.

Methods: this is an observational, prospective and unicentric trial including all coronary angioplasties performed in an interventional Cardiology center between October 2016 and January 2017, excluding instent reestenosis and coronary vein grafts disease. Two groups were defined: control (normal coronary flow) and no-reflow phenomenon. Clinical, angiographic and prognostic variables were analyzed.

Results: 322 patients were included, 291 control and 31 no-reflow. No-reflow incidence was 9.6\%, occurring in $20.7 \%$ of acute coronary syndromes with ST segment elevation, 6.3\% in non-ST segment elevation syndromes and 3\% in stable coronary artery disease. High dose adenosine was the most frequently used agent for no-reflow treatment. No-reflow treatment was associated with a significant improvement in coronary flow measured by TIMI Frame Count $(39.4 \pm 1.6$ pretreatment vs $21.9 \pm 1.2$ postreatment, $\mathrm{p}<0.001$ ). At 3 month follow up, no-reflow patients vs control had a higher incidence of angina pectoris ( $6.6 \%$ vs $1.1 \%$ respectively, $\mathrm{p}=0.001)$, coronary angiography ( $10 \%$ vs $1.1 \%, \mathrm{p}=0.017)$ and coronary angioplasty ( $19 \%$ vs $1.1 \%, \mathrm{p}=0.009)$.

Conclusions: no-reflow incidence in patients treated with coronary angioplasty was $9.6 \%$, occurring more frequently in ST segment elevation syndromes. High dose adenosine was the most frequently used agent for no-reflow treatment and significantly improved coronary flow.

Key words:

NO-REFLOW PHENOMENON
CORONARY ANGIOPLASTY
ADENOSINE
TIMI FRAME COUNT

\section{Introducción}

El fenómeno de no reflujo (NR) se define como la reducción del flujo coronario ( < TIMI 3) durante la realización de una angioplastia coronaria (ATC), sin evidencia angiográfica de obstrucción epicárdica, disección, espasmo o trombosis in situ, e implica una reducción aguda del flujo sanguíneo miocárdico e isquemia miocárdica consecuente $\mathrm{e}^{(1,2)}$.

El NR se presenta en hasta un $40 \%$ de los pacientes tratados con ATC primaria y en $<2 \%$ de los pacientes que se someten a intervencionismo electivo y ha sido reconocido como un predictor independiente de mal pronóstico ${ }^{(3)}$. En el contexto del sín-

Principales aportes

- Es el primer estudio nacional que analiza las características clínicas y angiográficas del fenómeno de no reflujo.

- Este fenómeno afecta a uno de cada diez procedimientos de angioplastia coronaria, predominantemente en el contexto de los síndromes coronarios agudos.

- Confirmamos la eficacia de la adenosina para la reversión del no reflujo.

- La asociación de este fenómeno con eventos clínicos adversos, sugerida por nuestros resultados, será motivo de futuras investigaciones con una mayor población de estudio. drome coronario agudo con elevación del segmento ST (SCAcST) se asocia con insuficiencia cardíaca y aumento de la mortalidad, independientemente del tamaño y localización del infarto ${ }^{(4,5)}$.

Existen predictores que pueden discriminar a pacientes de mayor riesgo de NR, como la edad avanzada, el infarto anterior y la hiperlipidemia(6). No está muy claro el papel de los agentes farmacológicos para la prevención del NR, pero existen medidas para el tratamiento del NR que incluyen tratamiento vasodilatador coronario (adenosina, nitroprusiato de sodio, nitroglicerina) y tratamiento antiagregante plaquetario, específicamente inhibidores GP IIb/IIIa.

Se consideró de interés conocer la incidencia de $\mathrm{NR}$ en nuestro medio en pacientes sometidos a ATC, los escenarios clínicos en los que se presenta, la respuesta al tratamiento instituido y su importancia pronóstica. Se destaca que no existen en nuestro medio estudios previos a este respecto.

\section{Material y método}

Realizamos un estudio observacional, prospectivo y unicéntrico, en el Servicio de Cardiología Intervencionista del Centro Cardiológico Americano (Sanatorio Americano). Fueron incluidos todos los pacientes sometidos a ATC sobre arterias nativas por 
cualquier indicación: SCAcST, síndrome coronario agudo sin elevación del segmento ST (SCAsST), ángor estable y estudios funcionales positivos. La firma de consentimiento informado fue requisito para la inclusión en el estudio. El período de inclusión fue de octubre de 2016 a enero de 2017.

Los criterios de exclusión fueron: procedimientos realizados sobre reestenosis intrastent y enfermedad de injertos venosos o mamarios.

Se definió el NR como la caída del flujo coronario durante la ATC, es decir, flujo TIMI $<3$ luego de una maniobra de desobstrucción coronaria en ausencia de obstrucción epicárdica. Esto permitió predefinir dos grupos: grupo control, con flujo coronario normal, y grupo NR, con fenómeno de NR.

El objetivo principal del estudio fue determinar la incidencia de NR en pacientes tratados con ATC por cualquier indicación. Los objetivos secundarios fueron describir las características clínicas y angiográficas de la población, las características del procedimiento de ATC, el tratamiento instituido para el NR y la respuesta al mismo, así como evaluar la importancia pronóstica del NR.

Las variables registradas incluyeron variables clínicas (edad, sexo, procedencia, indicación clínica del procedimiento de ATC), y variables del propio procedimiento (vaso tratado, número de stents implantados, flujo coronario inicial y ocurrencia de NR). El NR se evaluó a través de la valoración del flujo TIMI y a través del conteo de cuadros TIMI o TIMI Frame Count pre y postratamiento, tomando como referencia 27 cuadros o más como NR y menos de 27 como flujo normal ${ }^{(1)}$. Se registró el momento de aparición del NR respecto al procedimiento (luego de predilatación, de implante de stent o de posdilatación), el tiempo que el paciente permaneció en NR, así como el tratamiento instituido, la respuesta al mismo y sus complicaciones. Se evaluó la magnitud de la carga trombótica coronaria en base al score TIMI de carga trombótica (grado 0: ausencia de cualquier característica angiográfica de trombo; grado 1 : trombo posible, en base a reducción de la densidad de contraste, aspecto de vidrio esmerilado, lesión de contorno irregular o menisco convexo; grado 2: trombo definido, con longitud $<1 / 2$ del diámetro vascular; grado 3: trombo definido, con longitud $>1 / 2$ pero $<2$ diámetros vasculares; grado 4 : trombo definido con longitud $>2$ diámetros vasculares; grado 5 : oclusión trombótica total ${ }^{(7)}$. Se consideró alta carga trombótica los grados 4 y 5 . Se registraron variables pronósticas (estado sintomático, necesidad de nuevos procedimientos y mortalidad).

Las variables clínicas fueron obtenidas de la base de datos del Servicio de Hemodinamia del Centro Cardiológico Americano.
Las variables del procedimiento se obtuvieron de la planilla de registro de cada ATC así como de la evaluación de cada cineangiocoronariografia (CACG) para determinar la existencia o no de NR en forma independiente del equipo de cardiólogos intervencionistas actuante.

Los investigadores no intervinieron en el tratamiento del paciente y el mismo fue definido por el equipo médico actuante. No existió normatización de las dosis de fármacos intracoronarios.

Las variables pronósticas se obtuvieron en base a un seguimiento telefónico, según una planilla de interrogatorio predefinida.

Todas las variables referidas se ingresaron en una base de datos creada para este estudio.

\section{Análisis estadístico}

El análisis de los resultados se realizó en base a la comparación de los dos grupos predefinidos: grupo control y grupo NR. Se presentan tablas de frecuencia para la descripción de variables cualitativas; en el caso de las continuas se muestran medidas de resumen: media, desvío estándar, mínimos, máximos y mediana.

Se estudió la distribución normal de las variables continuas en estudio mediante test de Kolmogorov-Smirnov.

La incidencia acumulada se calculó en base al cociente entre el número de nuevos casos de NR y el total de pacientes en riesgo, definidos como todos los pacientes sometidos a ATC en el período de estudio. Para la comparación de variables continuas se utilizó el test $t$ de Student para muestras dependientes. Se consideró significativo un valor de $\mathrm{p} \leq 0,05$. El software estadístico utilizado correspondió a STATA v. 12.0.

Para el estudio de pronóstico se realizó un análisis bivariado, describiendo solo los valores de $\mathrm{p}$ de las comparaciones estadísticamente significativas.

Para garantizar los aspectos éticos de la investigación, los datos obtenidos fueron manejados con absoluta confidencialidad, con la autorización de cada paciente (firma de consentimiento informado) y del Comité de Ética del Sanatorio Americano. $\mathrm{El}$ investigador principal es el responsable de los datos.

\section{Resultados}

Este estudio incluyó un total de 322 pacientes consecutivos sometidos a ATC por cualquier indicación entre octubre de 2016 y enero de 2017 en el Servicio de Cardiología Intervencionista del Centro Cardiológico Americano. Los grupos predefinidos, control 


\begin{tabular}{|c|c|c|c|}
\hline Variable & Control $(n=291)$ & $N R(n=31)$ & $p$ \\
\hline \multirow{2}{*}{ Edad (media $\pm D E$, años) } & $62,7 \pm 11$ & $65,8 \pm 13$ & 0,235 \\
\hline & $n(\%)$ & $n(\%)$ & \\
\hline Sexo masculino & $225(77,3)$ & $26(83,9)$ & 0,689 \\
\hline HTA & $205(70,4)$ & $18(58,1)$ & 0,114 \\
\hline Tabaquismo & $82(28,2)$ & $12(38,7)$ & 0,154 \\
\hline Diabetes & $70(24,1)$ & $5(16,1)$ & 0,108 \\
\hline Dislipemia & $127(43,6)$ & $12(38,7)$ & 0,371 \\
\hline Obesidad & $31(10,7)$ & $4(12,9)$ & 0,443 \\
\hline Ángor estable & $32(11,0)$ & $1(3,2)$ & $0,029^{*}$ \\
\hline SCAsEST & $194(66,7)$ & $13(41,9)$ & 0,090 \\
\hline SCAcEST & $65(22,3)$ & $17(54,8)$ & $0,003^{*}$ \\
\hline
\end{tabular}

y NR, constaron de 291 y 31 pacientes respectivamente.

\section{Objetivo principal. Incidencia del fenómeno de no reflujo}

La incidencia de NR fue de 9,6\% (31 casos NR en 322 ATC) en la población total de pacientes sometidos a ATC.

\section{Objetivos secundarios}

Características clínicas de la población

La edad promedio de la población analizada fue de $63,1 \pm 11$ años, correspondiendo el $22 \%$ al sexo femenino. No se comprobaron diferencias significativas entre ambos grupos en la edad o en la incidencia de los factores de riesgo cardiovascular tradicionales (tabla 1), siendo la hipertensión arterial el factor de riesgo más frecuente en ambos grupos.

La forma de presentación más frecuente del NR fue el SCAcST (grupo NR 54,8\% vs grupo control $22,3 \%, p=0,003$ ). Se observó una distribución opuesta para la angina estable, que se presentó más frecuentemente en el grupo control en comparación con el grupo NR (grupo control 11,0\% vs grupo NR 3,2\%, $\mathrm{p}=0,029$ ). Si bien la incidencia de SCAsST fue numéricamente mayor en el grupo control que en el grupo NR, la diferencia no alcanzó significación estadística (grupo control 66,7\% vs grupo NR 41,9\%, $\mathrm{p}=0,09$ ).

Con respecto a la incidencia de NR, según las diferentes presentaciones clínicas que motivaron la ATC, fue máxima en el SCAcST, ocurriendo en el
Tabla 2. Incidencia de NR según la forma de presentación clínica.

\begin{tabular}{lccc}
\hline & Total $(n)$ & NR $(n)$ & $\%$ \\
\hline SCAcEST & 82 & 17 & 20,7 \\
SCAsEST & 207 & 13 & 6,3 \\
Ángor estable & 33 & 1 & 3,0 \\
\hline
\end{tabular}

NR: no reflujo; SCAcEST: síndrome coronario agudo con elevación de ST; SCAsEST: síndrome coronario agudo sin elevación de ST.

Tabla 3. Características del NR: tratamiento antiagregante.

\begin{tabular}{lc}
\hline & $n(\%)$ \\
\hline Aspirina & $31(100,0)$ \\
Clopidogrel $75 \mathrm{mg}$ & $2(6,5)$ \\
Clopidogrel $300 \mathrm{mg}$ & $20(64,5)$ \\
Clopidogrel $600 \mathrm{mg}$ & $4(12,9)$ \\
No clopidogrel & $5(16,1)$ \\
\hline
\end{tabular}

20,7\% de los casos, seguida por el SCAsST y la angina estable (tabla 2).

Se registró el tratamiento antiagregante previo a la ATC en el grupo de NR; la dosis carga de clopidogrel fue de $600 \mathrm{mg}$ en el 12,9\% de los casos y de $300 \mathrm{mg}$ en el $64,5 \%$ (tabla 3 ). 
Tabla 4. Características angiográficas de la población.

\begin{tabular}{lccc}
\hline Variable & $\begin{array}{c}\text { Control }(n=291) \\
n(\%)\end{array}$ & $\begin{array}{c}N R(n=31) \\
n(\%)\end{array}$ & $p$ \\
\hline Arteria ocluida & $65(22,3)$ & $19(61,3)$ & 0,002 \\
ADA & $124(42,6)$ & $10(32,3)$ & 0,279 \\
ACX & $62(21,3)$ & $11(35,5)$ & 0,100 \\
ACD & $105(36,1)$ & $10(32,2)$ & 0,298 \\
\hline
\end{tabular}

ADA: arteria descendente anterior; ACX: arteria circunfleja; ACD: arteria coronaria derecha

Tabla 5. Severidad de la enfermedad coronaria.

\begin{tabular}{lccc}
\hline Variables & $\begin{array}{c}\text { Control }(n=291) \\
n(\%)\end{array}$ & $\begin{array}{c}\text { NR }(n=31) \\
n(\%)\end{array}$ \\
\hline 1 vaso & $116(39,9)$ & $14(45,2)$ & $>0,05$ \\
2 vasos & $84(28,8)$ & $7(22,6)$ & 0,54 \\
3 vasos & $91(31,3)$ & $10(32,2)$ & $>0,05$ \\
\hline
\end{tabular}

Tabla 6. Características del procedimiento de angioplastia coronaria.

\begin{tabular}{|c|c|c|c|c|}
\hline & & $\begin{array}{c}\text { Control }(n=291) \\
n(\%)\end{array}$ & $\begin{array}{c}N R(n=31) \\
n(\%)\end{array}$ & $p$ \\
\hline Tromboaspiración & & $6(2,1)$ & $5(16,1)$ & 0,029 \\
\hline Predilatación & & $127(43,6)$ & $20(64,5)$ & 0,05 \\
\hline Implante directo de stent & & $148(50,9)$ & $0(0,0)$ & 0,001 \\
\hline Posdilatación & & $10(3,4)$ & $6(19,4)$ & 0,003 \\
\hline \multirow[t]{4}{*}{ Número de stents } & 1 & $229(78,7)$ & $16(51,6)$ & $>0,05$ \\
\hline & 2 & $55(18,9)$ & $10(32,3)$ & 0,001 \\
\hline & 3 & $6(2,1)$ & $4(12,9)$ & 0,001 \\
\hline & 4 & $1(0,3)$ & $1(3,2)$ & 0,001 \\
\hline
\end{tabular}

\section{Características angiográficas de la población}

En el grupo NR la mayoría de los pacientes presentó oclusión total inicial del vaso tratado (TIMI 0) y la diferencia con el grupo control fue estadísticamente significativa (NR 61,3\% vs control $22,3 \%, p=0,002$ ) (tabla 4).

No se encontró asociación entre NR y vaso culpable, encontrando en el grupo control mayor porcentaje de la arteria descendente anterior como vaso responsable y en el grupo NR mayor porcentaje de la arteria circunfleja (tabla 4), sin diferencias significativas entre grupos.
El análisis de la carga trombótica por score TIMI se limitó al grupo NR, comprobándose que el 67,8\% de los casos presentó alta carga trombótica.

En lo que respecta a la severidad de la enfermedad coronaria, evaluada en base al número de vasos afectados, no se comprobó diferencia significativa en la comparación entre grupos. En ambos grupos se observó un predominio de la lesión de 1 vaso (control: $39,9 \%$ vs NR: 45,2\%), seguido de lesión de 3 vasos (control: $31,3 \%$ vs NR: $32,2 \%$ ) y de lesión de 2 vasos (control: 28,8\% vs NR: 22,6\%) (tabla 5). 
Tabla 7. Relación de la aparición de NR con el procedimiento de angioplastia coronaria.

\begin{tabular}{lc}
\hline & $n(\%)$ \\
\hline Luego de predilatación & $5(16,1)$ \\
Luego de implante de stent & $26(83,9)$ \\
Luego de posdilatación & 0,0 \\
\hline
\end{tabular}

Características del procedimiento de angioplastia

La frecuencia de utilización de tromboaspiración, predilatación y posdilatación fue mayor en el grupo NR, con diferencia estadísticamente significativa con respecto al grupo control (tabla 6).

Respecto al uso de stents, el número de stents implantados por paciente fue mayor en el grupo NR (tabla 6).

En relación con el momento de presentación del NR, el mayor porcentaje ocurrió luego del implante de stents (tabla 7).

\section{Tratamiento del no reflujo}

El tratamiento farmacológico más frecuentemente utilizado para el NR fue la adenosina intracoronaria. Las dosis utilizadas de nitroglicerina (NTG) y adenosina corresponden a un amplio rango, con predominio de dosis elevadas, siendo las más frecuentes: NTG $400 \mu \mathrm{g}$ y adenosina $6 \mathrm{mg}$ por vía intracoronaria (tabla 8).

La eficacia del tratamiento farmacológico se evaluó mediante la comparación del recuento de cuadros TIMI o TIMI Frame Count antes y después del tratamiento. El TIMI Frame Count disminuyó significativamente luego del tratamiento farmacológico del NR, indicando mejoría del flujo epi-

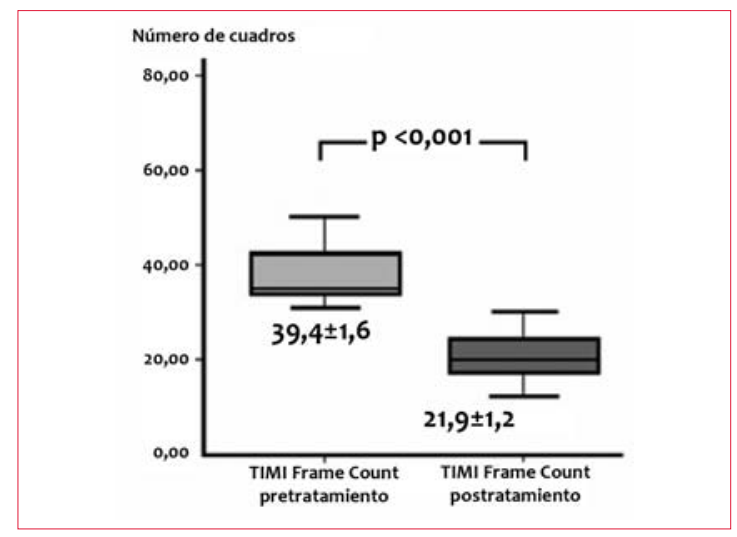

Figura 1. Eficacia del tratamiento del NR: TIMI Frame Count pre y postratamiento.

cárdico (pretratamiento: $39,4 \pm 1,6$ vs postratamiento: 21,9 $\pm 1,2, \mathrm{p}<0,001$ ) (figura 1).

Debe señalarse que en cinco casos $(16,1 \%)$ no se logró mejoría del flujo coronario.

Como dato adicional, el tiempo de recuperación promedio del flujo coronario fue de 4 minutos.

En los pacientes con NR persistente y alta carga trombótica se utilizó tirofibán (inhibidor GP IIb/IIIa) con dosis intracoronaria e intravenosa ajustada al peso del paciente en un total de tres pacientes, representando $9,6 \%$.

Con respecto a las complicaciones inmediatas con el uso de agentes intracoronarios se encontró que entre los tratados con NTG, tres pacientes presentaron hipotensión arterial transitoria, en tanto que entre los tratados con adenosina se observó bradicardia en once pacientes y bloqueo auriculoventricular completo transitorio en un paciente.

\section{Pronóstico del no reflujo}

La incidencia total de todos los eventos clínicos adversos intrahospitalarios registrados fue numéricamente mayor en el grupo NR, pero no fue posible

Tabla 8. Tratamiento del NR: agentes utilizados y dosis.

\begin{tabular}{|c|c|c|c|c|c|c|}
\hline & \multicolumn{2}{|c|}{ Adenosina* } & \multicolumn{2}{|c|}{ Nitroglicerina* } & \multicolumn{2}{|c|}{ Tirofibán** } \\
\hline & \multicolumn{2}{|r|}{$n(\%)$} & \multicolumn{2}{|r|}{$n(\%)$} & \multicolumn{2}{|r|}{$n(\%)$} \\
\hline & Total & $26(83,9)$ & Total & $10(32,2)$ & Total & $3(9,7)$ \\
\hline \multirow[t]{4}{*}{ Dosis } & $6 \mathrm{mg}$ & $17(65,4)$ & $200 \mu \mathrm{g}$ & $1(10,0)$ & \multicolumn{2}{|c|}{ Dosis por $\mathrm{kg}$ de peso } \\
\hline & $12 \mathrm{mg}$ & $7(27,0)$ & $300 \mu \mathrm{g}$ & $3(30,0)$ & & \\
\hline & $18 \mathrm{mg}$ & $1(3,8)$ & $400 \mu \mathrm{g}$ & $5(50,0)$ & & \\
\hline & $24 \mathrm{mg}$ & $1(3,8)$ & $500 \mu \mathrm{g}$ & $1(10,0)$ & & \\
\hline
\end{tabular}


Tabla 9. Complicaciones intrahospitalarias.

\begin{tabular}{lcc}
\hline & $\begin{array}{c}\text { Control }(n=291) \\
n(\%)\end{array}$ & $\begin{array}{c}\text { NR }(n=31) \\
n(\%)\end{array}$ \\
\hline Muerte & $1(0,3)$ & $1(3,2)$ \\
Ángor & 0 & $7(22,6)$ \\
Elevación del ST & 0 & $4(12,9)$ \\
$\begin{array}{l}\text { Repercusión } \\
\text { hemodinámica }\end{array}$ & 0 & $2(6,5)$ \\
Embolia distal & 0 & $2(6,5)$ \\
\hline
\end{tabular}

realizar un análisis estadístico comparativo por el bajo número de eventos (tabla 9 ).

Se realizó un seguimiento a tres meses a un total de 294 pacientes (91,3\%); en el 8,7\% restante no fue posible contactar a los pacientes. Respecto a los eventos, la incidencia de ángor, nueva internación, nueva CACG y nueva ATC del mismo vaso fue mayor en el grupo NR, con diferencia significativa respecto al grupo control. La incidencia total de eventos fue mayor en el grupo NR que en el grupo control (control $18[6,8 \%]$ vs $12[40 \%], p=0,001$ ) (tabla $10)$.

\section{Discusión}

El objetivo de la ATC es la recuperación del flujo coronario; sin embargo puede persistir una perfusión miocárdica inadecuada en ausencia de obstáculos en la circulación epicárdica, lo que se conoce como fenómeno de $\mathrm{NR}^{(8)}$.

Numerosos autores han demostrado una asociación significativa entre el NR y los eventos cardiovasculares adversos mayores, con incremento del riesgo de muerte, reinfarto y disfunción ventricular izquierda a corto y largo plazo(9-13). Esta importancia pronóstica es particularmente relevante en la ATC primaria en la que el NR se ha vinculado además a expansión del infarto(15-17).

El NR tiene una patogenia compleja que explica la dificultad para prevenirlo y tratarlo adecuadamente. Este fenómeno ha sido asociado a oclusión de la microcirculación coronaria, que reconocería una patogenia multifactorial, incluyendo microembolización de material aterotrombótico, vasoespasmo de la microcirculación y edema o hemorragia miocárdica (en el contexto de necrosis por infarto). Estos mecanismos se han asociado a activación de la cascada inflamatoria, liberación de radicales libres y agregación plaquetaria( ${ }^{(8,14)}$.

Múltiples maniobras del procedimiento intervencionista (tromboaspiración, implante directo de stent) así como múltiples agentes farmacológicos (adenosina, diltiazem, nicorandil, nitroprusiato de sodio, verapamil, adrenalina a bajas dosis) han sido utilizados en la prevención y el tratamiento del $\mathrm{NR}^{(8,14)}$.

El interés de realizar este estudio prospectivo y observacional se basó en determinar las características clínicas del NR en nuestro medio, donde no existen trabajos previos sobre este fenómeno.

\begin{tabular}{lccc} 
Tabla 10. Eventos en el seguimiento a tres meses. & & \\
\hline & $\begin{array}{c}\text { Control }(n=264) \\
n(\%)\end{array}$ & $\begin{array}{c}N R(n=30) \\
n(\%)\end{array}$ & 0,001 \\
\hline Ángor & $3(1,1)$ & $2(6,7)$ & - \\
Disnea & $2(0,8)$ & 0 & - \\
Nuevo infarto & $1(0,4)$ & 0 & - \\
Muerte & 0 & 0 & 0,050 \\
Nueva internación & $3(1,1)$ & $1(3,3)$ & 0,017 \\
Nueva CACG & $3(1,1)$ & $3(10,0)$ & 0,009 \\
Nueva ATC & $3(1,1)$ & $3(10,0)$ & 0,004 \\
$\quad 2(0,8)$ & $3(10,0)$ & - \\
$\quad 1(0,4)$ & 0 & - \\
Otro vaso & 0 & 0 & 0,001 \\
Mismo vaso & $18(6,8)$ & $12(40,0)$ & \\
\hline
\end{tabular}

CACG: cineangiocoronariografía; ATC: angioplastia coronaria; CRM: cirugía de revascularización miocárdica. 
Comprobamos una incidencia de NR de $9,6 \%$ en una población de pacientes sometidos a ATC de arterias coronarias nativas por cualquier indicación. La tendencia comprobada a una mayor edad en el grupo NR (65,8 vs 62,7 años) se correlaciona con evidencia de que la edad mayor de 60 años es un predictor independiente de $\mathrm{NR}^{(18)}$.

También existe evidencia de una mayor prevalencia de dislipemia y diabetes mellitus tipo 2 en el NR, sin embargo esto no se observó en nuestro estu$\operatorname{dio}^{(19,20)}$.

Se ha descrito que la intervención coronaria en el SCAcST se asocia a una mayor incidencia de $\mathrm{NR}^{(21)}$. En nuestra población la gran mayoría de casos de NR correspondió a síndromes coronarios agudos con o sin elevación del segmento ST. La incidencia de NR, según la presentación clínica que motivó la ATC, fue de $20,7 \%$ en SCAcST, 6,3\% en SCAsST y $3 \%$ en angina estable. Esta distribución coincide en general con la referida en la literatura, donde se señala una incidencia máxima en el contexto del infarto agudo de miocardio con elevación del ST, en el que alcanza hasta un $40 \%$ y mínima en el ángor estable, en el que es menor de $2 \%{ }^{(3)}$. Nuestros hallazgos ratifican que si bien el NR ocurre típicamente en los síndromes coronarios agudos, también puede presentarse en la enfermedad coronaria estable. En estos casos, su patogenia podría responder más probablemente a microembolización de material ateromatoso o a espasmo microvascular.

En lo que respecta al vaso culpable, se ha descrito que la arteria descendente anterior es un predictor de $\mathrm{NR}^{(21)}$. Sin embargo, en nuestro estudio no logramos demostrar una asociación significativa entre arteria culpable y NR, probablemente por el bajo número de casos incluidos.

El grupo NR presentó una elevada incidencia de alta carga trombótica y una mayor frecuencia de oclusión inicial del vaso culpable que el grupo control como indicador adicional de alta carga trombótica, la que ha sido asociada patogénicamente con el $\mathrm{NR}^{(21)}$.

Diversas maniobras han sido utilizadas durante la ATC para disminuir la carga trombótica o mejorar el resultado de la misma, incluyendo tromboaspiración, predilatación, implante de stent y posdilatación $^{(22-24)}$.

La tromboaspiración fue más frecuente en el grupo NR. Debe destacarse que en la práctica del centro donde se realizó el estudio, la tromboaspiración se utiliza según las recomendaciones de las guías, es decir de manera no sistemática ${ }^{(23)}$. La asociación comprobada no implica un efecto favorecedor de la tromboaspiración sobre el NR. Por el contrario, consideramos que la necesidad de realizar tromboaspiración es un indicador de alta carga trombótica y mayor riesgo de NR. Esta técnica se utiliza con el objetivo de disminuir dicha carga y así prevenir la embolia y el fenómeno de NR, lo que no siempre se logra.

La predilatación de la lesión a tratar resultó más frecuente entre los pacientes con NR y ha sido asociada patogénicamente con el mismo, probablemente porque esta maniobra favorece la embolia distal de material aterotrombótico ${ }^{(25)}$.

El implante de stents es un recurso universalmente aceptado en la ATC y su uso permite aumentar la tasa de éxito y disminuir complicaciones como la falla aguda de la ATC, en especial por trombosis o la reestenosis intrastent, particularmente para los stents farmacoactivos. La incidencia de implante directo de stent (sin insuflación previa de balón o predilatación) fue significativamente mayor en el grupo control vs NR $(50,8 \%$ vs $0 \%$, respectivamente), lo que podría vincularse a un efecto preventivo del implante directo de stent respecto al NR, evitando la predilatación, que como ya fue señalado, se ha vinculado al desarrollo de $\mathrm{NR}^{(25,26)}$.

La posdilatación del stent fue más frecuente en el grupo NR, esto podría explicarse porque al lograr una mejor aposición del stent, la posdilatación podría provocar microembolización distal del material aterotrombótico remanente ubicado entre la malla metálica y la pared arterial. Sin embargo, no comprobamos ningún caso de NR que se presentara luego de la posdilatación, lo que pone en duda el mecanismo propuesto.

El uso de múltiples stents se asoció a mayor incidencia de NR y esto podría vincularse a una mayor extensión de la placa de ateroma a tratar, con mayor riesgo de embolización distal.

Se ha demostrado que el tratamiento antitrombótico adecuado (anticoagulación y doble antiagregación plaquetaria) previo al procedimiento, reduce aproximadamente en $35 \%$ la incidencia de $\mathrm{NR}^{(31,32)}$. Encontramos que esta estrategia para la prevención del NR no se cumplió adecuadamente en nuestra serie, dado que la utilización de clopidogrel a dosis adecuada previa a la ATC fue infrecuente; esto constituye, sin dudas, un factor a corregir en el tratamiento de nuestros pacientes.

Con respecto al tratamiento del NR, encontramos que en el 93,5\% de los casos se recurrió al uso de vasodilatadores, siendo la adenosina el más frecuentemente utilizado. La adenosina es un nucleótido endógeno con múltiples efectos farmacológicos potencialmente útiles para la reversión del $\mathrm{NR}$, que incluyen vasodilatación, reducción de la concentración celular de calcio y de radicales libres y efecto antagonista de plaquetas y neutrófilos ${ }^{(8)}$. 
Múltiples estudios han evaluado el efecto de la adenosina para el tratamiento del NR. El estudio AMISTAD demostró la eficacia de la adenosina en el SCAcST luego de reperfusión con fibrinolíticos, reduciendo el tamaño del infarto ${ }^{(33)}$. Marzilli y colaboradores demostraron que la adenosina mejora el flujo coronario con reducción de eventos cardíacos adversos luego de la angioplastia coronaria(34). Sdringola y colaboradores demostraron que altas dosis de adenosina intracoronaria son eficaces para revertir el NR en la ATC de puentes venosos ${ }^{(35)}$.

Más recientemente, Niccoli y colaboradores realizaron un estudio randomizado para valorar el efecto de la adenosina y del nitroprusiato de sodio luego de la tromboaspiración para la prevención de la oclusión microvascular en pacientes con SCAcST tratados con ATC primaria. Sobre 240 pacientes, compararon adenosina, nitroprusiato de sodio o placebo luego de la tromboaspiración. Se comprobó que la adenosina, pero no el nitroprusiato, se asoció con mayor incidencia de resolución completa del segmento ST, de flujo TIMI 3, de blush miocárdico TIMI 3 y menor incidencia de eventos cardiovasculares adversos ${ }^{(27)}$.

Tres metaanálisis recientes permiten concluir que la adenosina intracoronaria utilizada en pacientes con SCAcST tratados con ATC primaria se asocia con menor incidencia de flujo TIMI $<3$ y mayor frecuencia de renivelación del segmento $\mathrm{ST}^{(28-30)}$. El metaanálisis de red de Niu y colaboradores evaluó el efecto de siete agentes farmacológicos de uso intracoronario (adenosina, anisodamina, diltiazem, nicorandil, nitroprusiato, urapidil y verapamil) sobre el NR en pacientes con SCAcST tratados con ATC primaria. En lo que se refiere a la adenosina, si bien este metaanálisis comprobó mejoría del flujo coronario, no demostró mejoría de la fracción de eyección ventricular izquierda, ni disminución de efectos cardiovasculares adversos con el uso de la adenosina(30). Estos resultados negativos del metaanálisis podrían justificarse por la inclusión del estudio randomizado REFLO-STEMI, que comparó la eficacia de dosis consideradas como altas de adenosina $(2-3 \mathrm{mg})$ versus nitroprusiato $(500 \mu \mathrm{g})$ para reducir el tamaño del infarto o la oclusión microvascular determinados por resonancia magnética cardíaca. Contrariamente a lo esperado, no se comprobó reducción del tamaño del infarto o de la incidencia de oclusión microvascular con ninguna de las estrategias terapéuticas y la adenosina se asoció a un aumento de los eventos adversos y a una reducción de la fracción de eyección a los seis meses, aunque este último efecto fue moderado(31).

En definitiva, parece razonable aceptar, a la luz de la evidencia disponible, que la adenosina mejora el flujo coronario y la perfusión miocárdica en el NR, especialmente en los SCAcST tratados con ATC primaria, persistiendo un margen de duda sobre su efecto sobre la función ventricular y sobre la posibilidad de efectos cardiovasculares adversos a mediano plazo.

Existen nuevas opciones terapéuticas para el NR, como la anisodamina (agente antimuscarínico, bloqueador de sobrecarga celular de calcio, de la superoxidación lipídica y de la formación de radicales libres), que ha demostrado mejorar el flujo coronario epicárdico y la resolución del segmento ST, así como la fracción de eyección del ventrículo izquierdo y reducir eventos cardiovasculares adversos, como lo demuestra el metaanálisis de Niu y colaboradores $^{(30)}$. En este metaanálisis sobre 42 estudios randomizados y 4.069 pacientes, la anisodamina mostró la mejor performance entre siete drogas estudiadas, incluyendo la adenosina.

Existen varios estudios que respaldan el uso de inhibidores GP IIb/IIIa intraprocedimiento con el objetivo de disminuir la carga trombótica y aumentar la reperfusión microvascular después de la intervención coronaria ${ }^{(37-39)}$. En nuestro estudio se recurrió infrecuentemente al uso de inhibidores GP IIb/IIIa (9,6\% de los casos) y siempre en combinación con un vasodilatador coronario, por lo que no fue posible evaluar su eficacia. Con respecto a la nitroglicerina, no hay evidencia contundente que apoye su beneficio ${ }^{(40)}$, siendo su efecto vasodilatador coronario la base racional para su aplicación en el NR.

Las dosis más frecuentemente utilizadas de vasodilatadores intracoronarios en nuestro estudio fueron $6 \mathrm{mg}$ para adenosina y $400 \mu \mathrm{g}$ para nitroglicerina. Las dosis de adenosina utilizadas en estudios previos estuvieron en torno a $120 \mu \mathrm{g}$ por vía intracoronaria, en algunos casos seguidos de $3 \mathrm{mg}$ por vía intravenosa, aunque también se ha demostrado beneficio con dosis mayores ${ }^{(27)}$. En cualquier caso, las dosis utilizadas en nuestra serie deben ser consideradas como altas.

La eficacia del tratamiento vasodilatador coronario durante la intervención se puso en evidencia a través del TIMI Frame Count, aplicado a todos los casos de NR. Por este método pudimos demostrar una mejoría estadísticamente significativa en el flujo coronario epicárdico en forma objetiva, puesta en evidencia por una reducción promedio de $44 \%$ aproximadamente en el TIMI Frame Count posprocedimiento. Como contrapartida, el tratamiento fue ineficaz en cinco pacientes $(16,1 \%)$.

Las complicaciones del NR intraprocedimiento e intrahospitalarias son bien conocidas e incluyen persistencia de la sobreelevación del ST, arritmias y 
aumento de la mortalidad de hasta tres veces para mortalidad intrahospitalaria(41-45). También se ha asociado el NR a mayor incidencia de accidente cerebrovascular ${ }^{(45,46)}$ y de embolia distal del vaso a tra$\operatorname{tar}^{(47)}$. En nuestra serie no comprobamos diferencias significativas en la incidencia de eventos cardiovasculares adversos intrahospitalarios entre grupos, probablemente por el tamaño reducido de la muestra.

Sin embargo, en el seguimiento a tres meses en el grupo NR la probabilidad de recibir una nueva CACG fue 8,3 veces mayor y la de recibir una nueva ATC fue 13 veces mayor ( 27 veces mayor para ATC en otro vaso comparado con el grupo control). La incidencia de enfermedad multivaso fue similar en los dos grupos, por lo que la mayor incidencia de nueva revascularización no puede ser explicada por esta característica. Podría existir un vínculo entre NR y la necesidad de nueva revascularización a través de un fenómeno inflamatorio desencadenado por el $\mathrm{NR}$, que afectara placas vulnerables preexistentes.

\section{Limitaciones}

Debe señalarse que se trata de un estudio observacional, unicéntrico, en el que no se intervino en el tratamiento del paciente. Este fue definido por el equipo médico actuante, lo que puede generar sesgos en la elección de la estrategia terapéutica. No existió normatización de las dosis de fármacos intracoronarios, lo que si bien constituye una limitación metodológica, refleja la práctica habitual de un solo centro de cardiología intervencionista.

El tamaño reducido de la muestra, el corto período de seguimiento, así como la ausencia de registro de algunas variables como pico enzimático pos-ATC, evaluación ecocardiográfica y detección de arritmias, constituyeron limitaciones para evaluar el pronóstico a corto y largo plazo de nuestra población.

\section{Conclusiones}

El NR se presentó en uno de cada diez pacientes sometidos a ATC por cualquier motivo y fue más frecuente en el SCAcST, en pacientes con arteria ocluida, con alta carga trombótica, implante de múltiples stents y posdilatación del stent. El tratamiento vasodilatador coronario, en especial la adenosina a altas dosis, fue eficaz para mejorar el flujo coronario. Este estudio debe ser considerado como una primera etapa de investigación del NR, que deberá continuar con un estudio adecuadamente dimensionado en cuanto al tamaño de la muestra y el período de seguimiento.

\section{Agradecimientos}

Agradecemos a todo el grupo médico y de enfermería del Servicio de Hemodinamia y Cardiología Intervencionista del Centro Cardiológico Americano por su colaboración altamente calificada durante la investigación.

\section{Bibliografía}

1. Gibson C, Cannon C, Daley W, Dodge JT Jr, Alexander B Jr, Marble S, et al. TIMI frame count: a quantitative method of assessing coronary artery flow. Circulation 1996; 93(5):879-88.

2. Hamada S, Nishiue T, Nakamura S, Sugiura T, Kamihata H, Miyoshi H, et al. TIMI Frame Count immediately after primary coronary angioplasty as a predictor of functional recovery in patients with TIMI 3 reperfused acute myocardial infarction. J Am Coll Cardiol 2001; 38(3): 666-71.

3. Rezkalla S, Kloner R. Coronary no-reflow phenomenon: from the experimental laboratory to the cardiac catheterization laboratory. Catheter Cardiovasc Interv 2008; 72(7):950-7.

4. Nallamothu B, Bradley E, Krumholz H. Time to treatment in primary percutaneous coronary intervention. N Engl J Med 2007; 357(16):1631-8.

5. Niccoli G, Burzotta F, Galiuto L, Crea F. Myocardial no-reflow in humans. J Am Coll Cardiol 2009; $54(4): 281-92$

6. Claessen B, Kikkert W, Engstrom A, Hoebers L, Damman P, Vis M, et al. Primary percutaneous coronary intervention for ST elevation myocardial infarction in octogenarians: trends and outcomes. Heart 2010; 96(11):843-7.

7. Gibson C, de Lemos J, Murphy S, Marble S, McCabe C, Cannon C, et al. Combination therapy with abciximab reduces angiographically evident thrombus in acute myocardial infarction: a TIMI 14 substudy. Circulation 2001; 103(21):2550-4.

8. Feher A, Chen S, Bagi Z, Arora V. Prevention and treatment of no-reflow phenomenon by targeting the coronary microcirculation. Rev Cardiovasc Med 2014; 15(1):38-51.

9. Ndrepepa G, Tiroch K, Keta D, Fusaro M, Seyfarth M, Pache J, et al. Predictive factors and impact of no reflow after primary percutaneous coronary intervention in patients with acute myocardial infarction. Circ Cardiovasc Interv 2010; 3(1):27-33

10. Dong-bao L, Qi H, Zhi L, Shan W, Wei-ying J. Predictors and long-term prognosis of angiographic slow/no-reflow phenomenon during emergency percutaneous coronary intervention for ST-elevated acute myocardial infarction. Clin Cardiol 2010; 33(12):E7-12. 
11. Rezkalla S, Dharmashankar K, Abdalrahman I, Kloner R. No-reflow phenomenon following percutaneous coronary intervention for acute myocardial infarction: incidence, outcome, and effect of pharmacologic therapy. J Interv Cardiol 2010; 23(5): 429-36.

12. Iwakura K, Ito H, Kawano S, Shintani Y, Yamamoto K, Kato A, et al. Predictive factors for development of the no-reflow phenomenon in patients with reperfused anterior wall acute myocardial infarction. J Am Coll Cardiol 2001; 38(2):472-7.

13. Ndrepepa G, Tiroch K, Fusaro M, Keta D, Seyfarth M, Byrne R, et al. 5-year prognostic value of no-reflow phenomenon after percutaneous coronary intervention in patients with acute myocardial infarction. J Am Coll Cardiol 2010; 55(21):2383-9.

14. Jaffe R, Charron T, Puley G, Dick A, Strauss B. Microvascular obstruction and the no-reflow phenomenon after percutaneous coronary intervention. Circulation 2008; 117(24):3152-6.

15. Hamirani Y, Wong A, Kramer C, Salerno M. Effect of microvascular obstruction and intramyocardial hemorrhage by CMR on LV remodeling and outcomes after myocardial infarction: a systematic review and meta-analysis. JACC Cardiovasc Imaging 2014; 7(9):940-52.

16. Sattur S, Sarwar B, Sacchi T, Brener S. Correlation between markers of reperfusion and mortality in ST-elevation myocardial infarction: a systematic review. J Invasive Cardiol 2014; 26(11):587-95.

17. Niccoli G, Cosentino N, Spaziani C, Loria V, Fracassi F, Roberto M, et al. Concordance of angiographic and electrocardiographic indexes of microvascular obstruction: myocardial haemorrhage role. J Cardiovasc Med (Hagerstown) 2016; 17(5): $382-91$.

18. Kirma C, Izgi A, Dundar C, Tanalp A, Oduncu V, Aung S, et al. Clinical and procedural predictors of no-reflow phenomenon after primary percutaneous coronary interventions: experience at a single center. Circ J 2008; 72(5):716-21.

19. Collet J, Montalescot G. The acute reperfusion management of STEMI in patients with impaired glucose tolerance and type 2 diabetes. Diab Vasc Dis Res 2005; 2(3):136-43.

20. Golino P, Maroko P, Carew T. The effect of acute hypercholesterolemia on myocardial infarct size and the no-reflow phenomenon during coronary occlusion-reperfusion. Circulation 1987; 75(1):292-8.

21. Cura F, García Escudero A, Berrocal D, Mendiz O, Albertal M, Baccaro J, et al. Predictores de reperfusión miocárdica tisular luego de la angioplastia en el infarto agudo de miocardio. Rev Argent Cardiol $2009 ; 77(3): 174-80$
22. Fröbert O, Lagerqvist B, Olivecrona G, Omerovic E, Gudnason T, Maeng M, et al. Thrombus aspiration during ST-segment elevation myocardial infarction. N Engl J Med 2013; 369(17):1587-97.

23. Levine G, Bates E, Blankenship J, Bailey S, Bittl J, Cercek B, et al. 2015 ACC/AHA/SCAI focused update on primary percutaneous coronary intervention for patients with ST-elevation myocardial infarction: an update of the 2011 ACCF/AHA/SCAI guideline for percutaneous coronary intervention and the $2013 \mathrm{ACCF} / \mathrm{AHA}$ guideline for the management of ST-elevation myocardial infarction. J Am Coll Cardiol 2016; 67(10):1235-1250. doi: 10.1016/j.jacc.2015. 10.005

24. Stone G, Maehara A, Witzenbichler B, Godlewski J, Parise H, Dambrink J, et al. Intracoronary abciximab and aspiration thrombectomy in patients with large anterior myocardial infarction: the INFUSE-AMI randomized trial. JAMA 2012; 307(17):1817-26

25. Piscione F, Piccolo R, Cassese S, Galasso G, D'Andrea C, De Rosa R, et a. Is direct stenting superior to stenting with predilation in patients treated with percutaneous coronary intervention? Results from a meta-analysis of 24 randomised controlled trials. Heart 2010; 96(8):588-94.

26. Vlaar P, Svilaas T, van der Horst I, Diercks G, Fokkema M, de Smet B, et al. Cardiac death and reinfarction after 1 year in the Thrombus Aspiration during Percutaneous coronary intervention in Acute myocardial infarction Study (TAPAS): a 1-year follow-up study. Lancet 2008; 371(9628):1915-20.

27. Niccoli G, Rigattieri S, De Vita M, Valgimigli M, Corvo P, Fabbiocchi F, et al. Open-label, randomized, placebo-controlled evaluation of intracoronary adenosine or nitroprusside after thrombus aspiration during primary percutaneous coronary intervention for the prevention of microvascular obstruction in acute myocardial infarction: the REOPENAMI study (Intracoronary Nitroprusside Versus Adenosine in Acute Myocardial Infarction). JACC Cardiovasc Interv 2013; 6(6):580-9.

28. Polimeni A, De Rosa S, Sabatino J, Sorrentino S, Indolfi C. Impact of intracoronary adenosine administration during primary PCI: A meta-analysis. Int J Cardiol 2016; 203:1032-41.

29. Gao Q, Yang B, Guo Y, Zheng F. Efficacy of adenosine in patients with acute myocardial infarction undergoing primary percutaneous coronary intervention: a PRISMA-Compliant meta-analysis. Medicine (Baltimore) 2015; 94(32):e1279.

30. Niu X, Zhang J, Bai M, Peng Y, Sun S, Zhang Z. Effect of intracoronary agents on the no-reflow phenomenon during primary percutaneous coronary intervention in patients with ST-elevation myocardial 
infarction: a network meta-analysis. BMC Cardiovasc Disord 2018; 18(1):2-15.

31. Nazir S, McCann G, Greenwood J, Kunadian V, Khan J, Mahmoud I, et al. Strategies to attenuate micro-vascular obstruction during P-PCI: the randomized reperfusion facilitated by local adjunctive therapy in ST-elevation myocardial infarction trial. Eur Heart J 2016; 37(24):1910-9.

32. Jung J, Min P, Lee S, Sung C, Choi S, Cho J, et al. Clopidogrel pretreatment before primary percutaneous coronary stenting in patients with acute ST-segment elevation myocardial infarction: comparison of high loading dose $(600 \mathrm{mg})$ versus low loading dose (300 mg). Coron Artery Dis 2009; 20(2):150-4.

33. Patti G, Colonna G, Pasceri V, Pepe L, Montinaro A, Di Sciascio G. Randomized trial of high loading dose of clopidogrel for reduction of periprocedural myocardial infarction in patients undergoing coronary intervention: results from the ARMYDA-2 (Antiplatelet therapy for Reduction of MYocardial Damage during Angioplasty) study. Circulation 2005; 111(16):2099-106

34. Mahaffey K, Puma J, Barbagelata N, DiCarli M, Leesar M, Browne K, et al. Adenosine as an adjunct to thrombolytic therapy for acute myocardial infarction: results of a multicenter, randomized, placebo-controlled trial: the Acute Myocardial Infarction STudy of Adenosine (AMISTAD) trial. J Am Coll Cardiol 1999; 34(6):1711-20.

35. Marzilli M, Gliozheni E, Marraccini P, Fedele S. Primary coronary angioplasty in acute myocardial infarction: clinical correlates of the 'no reflow' phenomenon. Int J Cardiol 1998; 65(Suppl 1):S23-8.

36. Sdringola S, Assali A, Ghani M, Yepes A, Rosales O, Schroth G, et al. Adenosine use during aortocoronary vein graft interventions reverses but does not prevent the slow-no reflow phenomenon. Catheter Cardiovasc Interv 2000; 51(4):394-9.

37. De Luca G, Suryapranata H, Piscione F, Barbato E, Chiariello M. Stenting and abciximab in primary angioplasty: a review of current status. Ital Heart J 2005; 6(11):874-80.

38. Montalescot G, Antoniucci D, Kastrati A, Neumann F, Borentain M, Migliorini A, et al. Abciximab in primary coronary stenting of ST-elevation myocardial infarction: a European meta-analysis on individual patients' data with long-term follow-up. Eur Heart J 2007; 28(4):443-9.

39. De Luca G, Suryapranata H, Stone G, Antoniucci $D$, Tcheng J, Neumann F, et al. Abciximab as adjunctive therapy to reperfusion in acute ST-segment elevation myocardial infarction: a meta-analysis of randomized trials. JAMA 2005; 293(14): 1759-65.

40. Butler M, Chan W, Taylor A, Dart A, Duffy S. Management of the no-reflow phenomenon. Pharmacol Ther 2011; 132(1):72-85.

41. Yellon D, Hausenloy D. Myocardial reperfusion injury. N Engl J Med 2007; 357(11):1121-35.

42. Matsumura K, Jeremy R, Schaper J, Becker L. Progression of myocardial necrosis during reperfusion of ischemic myocardium. Circulation 1998; 97(8):795-804.

43. Gressin V, Louvard Y, Pezzano M, Lardoux H. Holter recording of ventricular arrhythmias during intravenous thrombolysis for acute myocardial infarction. Am J Cardiol 1992; 69(3):152-9.

44. Heper G, Korkmaz M, Kilic A. Reperfusion arrhythmias: are they only a marker of epicardial reperfusion or continuing myocardial ischemia after acute myocardial infarction? Angiology 2007; 58(6):663-70

45. Budaj A, Flasinska K, Gore J, Anderson F Jr, Dabbous O, Spencer F, et al. Magnitude of and risk factors for in-hospital and postdischarge stroke in patients with acute coronary syndromes: findings from a Global Registry of Acute Coronary Events. Circulation 2005; 111(24):3242-7.

46. Guptill J, Mehta R, Armstrong P, Horton J, Laskowitz D, James S, et al. Stroke after primary percutaneous coronary intervention in patients with ST-segment elevation myocardial infarction: timing, characteristics, and clinical outcomes. Circ Cardiovasc Interv 2013; 6(2):176-83.

47. Henriques J, Zijlstra F, Ottervanger J, de Boer $\mathbf{M}$, van 't Hof A, Hoorntje J, et al. Incidence and clinical significance of distal embolization during primary angioplasty for acute myocardial infarction. Eur Heart J 2002; 23(14):1112-7. 